\title{
Catalysis by nature
}

\author{
Welcome to the first issue of Nature Catalysis. While the format of a Nature Research journal will probably be \\ familiar to most of our readers, in this editorial we would like discuss the unique aspects of this journal and our \\ aims for the future.
}

P erhaps no area of chemistry has such a huge impact on society as catalysis. While this may (unfortunately) be a surprise to a significant percentage of the lay public, it is almost a truism for people knowledgeable of the field. Catalysis is fundamental to numerous endeavours including making the consumer products we use every day, producing the drugs that keep the population healthy, helping agriculture feed the planet, breaking down environmental pollutants in air and water, and generating, storing and converting energy. Some of these areas, in addition to the topics that catalysis research needs to focus on in the future, are covered very nicely by Sir John Meurig Thomas in his Comment in this issue.

Owing to this extraordinary range of applications, Nature Catalysis will not only cover the basic sciences, but also accordingly provide coverage of advances of an applied nature. While scientific excellence is always the primary criteria, work that reaches beyond the lab and influences everyday life - or is likely to inform processes that do so - is particularly interesting for us. As such, the importance of applied work will be reflected in both our research and comment sections - indeed, given the aforementioned effects that catalysis has on society, it would be surprising if this were not the case.

That is not to say that fundamental advances will be overlooked - on the contrary, we recognize that fundamental and applied work rely intimately on each other. To quote George Porter: "To feed applied science by starving basic science is like economizing on the foundations of a building so that it may be built higher. It is only a matter of time before the whole edifice crumbles."

(New Scientist 111, 16; 1986). In this, as in most things, a balance is needed and the diverse reports in Nature Catalysis will reflect the reality of the diverse range of research that makes up the catalysis community.

\section{While scientific excellence is always the primary criteria, work that reaches beyond the lab and influences everyday life - or is likely to inform processes that do so - is particularly interesting for us.}

So what do we aim to achieve with the journal? Catalysis is a field with a huge scope, from the study of fundamental physical properties to genetically engineering chemical-producing microorganisms. For any one person to be an expert in all these areas is, to put it mildly, a challenge, and even keeping up with the literature in subsets of catalysis is not easy. Yet evermore exciting developments are appearing in the overlaps between traditionally established disciplines. Nature Catalysis aims to publish papers of broad interest that will not just interest researchers in that specific field - though they will of course remain the primary audience - but also be informative for people in nominally different areas of catalysis. The analysis and commentary articles for which Nature Research journals are known (for example, the News \& Views and Comments that you will find in this issue) will further help in bringing research breakthroughs to a wider audience. In this way, we hope that the journal may become a home for people to stay up to date with major advances in their own field, and also keep abreast of exciting developments in the broader catalysis community. Furthermore, as outlined above, it will be a forum that brings together the fundamental and applied scientist.

Of course, we hope that our readers also become contributors to the journal. As an author, you can rest assured that your work will reach a wide audience of scientists. The same procedures that ensure the work is of interest to a broad audience will help disseminate your breakthroughs to the wider catalysis community and beyond.

Finally, a brief note on the editorial team. We are all housed in the new Berlin office, and together provide expertise across the range of catalysis research. Enda Bergin joined the journal after managing one of the chemistry teams at Nature Communications, having previously lectured and ran a research group in homogeneous catalysis. Davide Esposito - another former principal investigator - joined us from the Max Planck Institute for Colloids and Interfaces, bringing up-todate knowledge in both homogeneous and heterogeneous systems. Marçal CapdevilaCortada has a strong background in computational catalysis, having most recently worked in this area at the Institute of Chemical Research of Catalonia (ICIQ), and will handle computational and materials content. Jan-Stefan Völler, who has joined Nature Catalysis from the Technical University of Berlin, is your point of contact for work on biocatalysis and related areas.

As a team, we very much look forward to working with you, and to seeing first-hand how catalysis continues to impact and shape our society.

Published online: 8 January 2018 https://doi.org/10.1038/s41929-017-0020-2 